\title{
A Digital Heterodyne 2-150 kHz Measurement Method
}

This paper was downloaded from TechRxiv (https://www.techrxiv.org).

\section{LICENSE}

CC BY 4.0

SUBMISSION DATE / POSTED DATE

06-01-2021 / 06-01-2021

\section{CITATION}

Wright, Paul; Ritzmann, Deborah (2021): A Digital Heterodyne 2-150 kHz Measurement Method. TechRxiv. Preprint. https://doi.org/10.36227/techrxiv.13525592.v1

$\mathrm{DOI}$ 


\title{
A Digital Heterodyne 2-150 kHz Measurement Method
}

\author{
P. S. Wright ${ }^{*}$ and D. Ritzmann* \\ *National Physical Laboratory, UK \\ paul.wright@npl.co.uk
}

\begin{abstract}
This paper describes a new method for measurements of signals in the $2-150 \mathrm{kHz}$ frequency range, as required to support the regulation of conducted emissions on the power grid. The digital method is based on heterodyning, decimation and multi resolution analysis.

Index Terms - Digital Filters, digital signal processing, electromagnetic compatibility and interference, power quality, quadrature amplitude modulation.
\end{abstract}

\section{INTRODUCTION}

The measurement of signals in the $2-150 \mathrm{kHz}$ range has become important due to the build-up of emissions on the power grid caused by power convertors such as those used in distributed generation [1]. Radio standard CISPR16-1 [2] specifies a spectral sweep method using an analogue tuned receiver. An equivalent real-time digital method is required that can be used in power quality instrumentation such that $2-150 \mathrm{kHz}$ conducted emissions on the grid can be accurately measured and regulated. This paper describes a proposed method based on a digital super heterodyne.

\section{DESCRIPTION OF THE MEASUREMENT METHOD}

The proposed digital method is based on three signal processing methods, namely heterodyning, multi-resolution analysis and decimation.

\section{A. Heterodyning}

Heterodyning [3] as used in analogue or digital radio receivers, takes a frequency band centred on a given frequency and shifts it to a new centre frequency using a multiplying mixer with an oscillator frequency $\left(f_{h}\right)$. This creates two new signals at the sum and difference of the two frequencies. As seen in Fig.1, to select a bandwidth $f_{b}$ centred on $f_{h}$, the oscillator frequency is set to $f_{h}$, and the resulting difference frequency will be centred on $0 \mathrm{~Hz}$, A low-pass filter (LPF) can then be used to select the required bandwidth $\mathrm{f}_{\mathrm{b}}$. For example, to measure all the frequency components between $2 \mathrm{kHz}$ and $2.2 \mathrm{kHz}, \mathrm{f}_{\mathrm{h}}$ would be set to $2.1 \mathrm{kHz}$ and the bandwidth of the LPF would be set to $100 \mathrm{~Hz}$. The resulting heterodyned band centred around $0 \mathrm{~Hz}$ between $-100 \mathrm{~Hz}$ and $+100 \mathrm{~Hz}$ would remain and all other frequencies, including the sum, will be attenuated by the LPF. F $F_{s}$ in Fig.1, is the sampling frequency of the analogue to digital converter (ADC) and "images" produced by the heterodyne process are also attenuated by the LPF.

In the context of this application, multiple heterodynes could be used with different $f_{h}$ mixer frequencies, to shift each frequency band of interest in the $2-150 \mathrm{kHz}$ band down to $0 \mathrm{~Hz}$. To use heterodynes to divide $2-150 \mathrm{kHz}$ into $200 \mathrm{~Hz}$ wide bands, would require 741 heterodynes with associated LPFs.

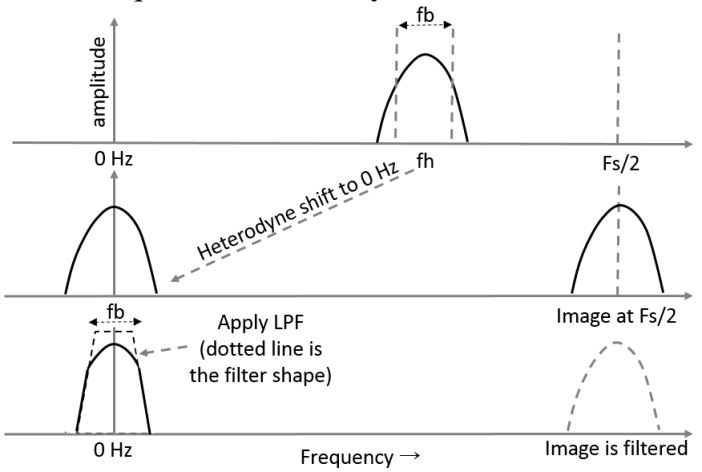

Fig. 1 Heterodyne spectrum with a band shift to $0 \mathrm{~Hz}$ and filtering.

The accuracy of the method is determined by the LPF cut-off which should be as sharp as possible, implying a high-order LPF. The digital LPF processes data at a rate of at least 2 times $150 \mathrm{kHz}$, which requires considerable processing and real-time measurement is unlikely to be feasible with multiple LPFs.

\section{B. Multi-Resolution Analysis (MRA)}

MRA [4] was devised for use with wavelets and is effectively a hierarchy of bandpass filters that break the measurement spectrum into a tree structure, dividing the bandwidth at each level of the tree. The MRA divided spectrum is shown in Fig. 2.

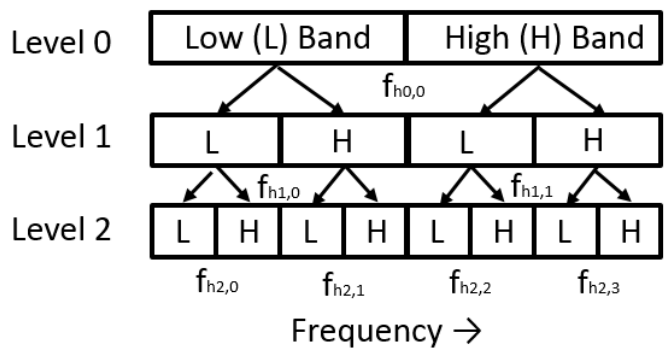

Fig. 2 MRA Tree progressively dividing the frequency band at each level. $f_{\mathrm{hl}, \mathrm{b}}$ are the heterodyne frequencies used at each level and band.

MRA analysis using wavelets has been applied to $2-150 \mathrm{kHz}$ measurements in [5], in a similar way heterodynes can use a classical LPF at each level to perform the MRA.

\section{Decimation}

The LPF processing speed can be improved by reducing the sample rate, but this is not allowed under the Nyquist restrictions. However, after the LPF, the signal bandwidth is 
reduced and down-sampling can be used to reduce the computational burden of subsequent MRA levels (there are less samples to process) and also allows the low pass filter specification to be relaxed at lower levels. This classical multirate signal processing approach can give a significant processing speed advantage in this application.

\section{Heterodyne based MRA measurement method}

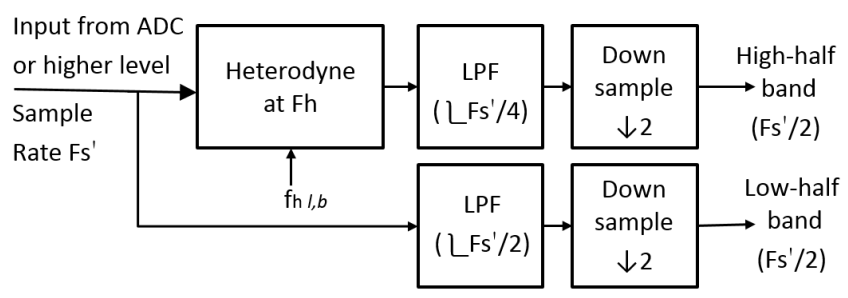

Fig. 3 One modular element of the MRA method applied at frequency $f_{h} l, b$. Input is a sampled waveform at sample rate Fs'; output is high and low band-limited sampled waveforms at $\mathrm{Fs}^{\prime} / 2$.

Fig.3 shows how each element of the low and high pass operation can be achieved with a heterodyne and LPFs. This MRA modular element takes input samples (at $F_{s}{ }^{\prime}$ sampling rate) and splits the bandwidth into low and high parts, outputting two sample sequences at half the sampling rate of the input. These MRA elements are identical for the whole processing tree, including the filter break points. The modular nature is convenient as the designer can concentrate effort into optimising the accuracy vs. computational speed of the module.

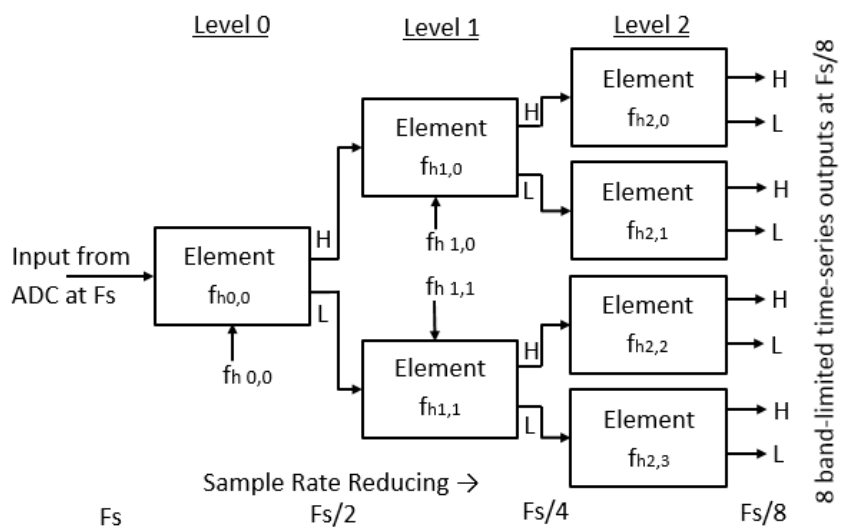

Fig. 4 Illustrative 3-level MRA using the connected modular elements shown in Fig. 3.

The MRA elements are connected together as shown in Fig.4. In this illustrative example with three levels, the spectrum is split into eight bands at the output of level 2. Note that the sampling rate is halved after each level, so although the number of elements doubles at each level, the amount of data that needs to be processed by each MRA element halves. The frequency response of the MRA method is shown in Fig.5 which plots amplitude response against frequency for each level. At level 0 in Fig.5, the high and the low band output response can be seen with the transition at half way at Fs/4 where the upper band is limited to Fs/2 as seen on the X-axis. The output at level 2, gives the signal split into 8 bands for post processing and display.

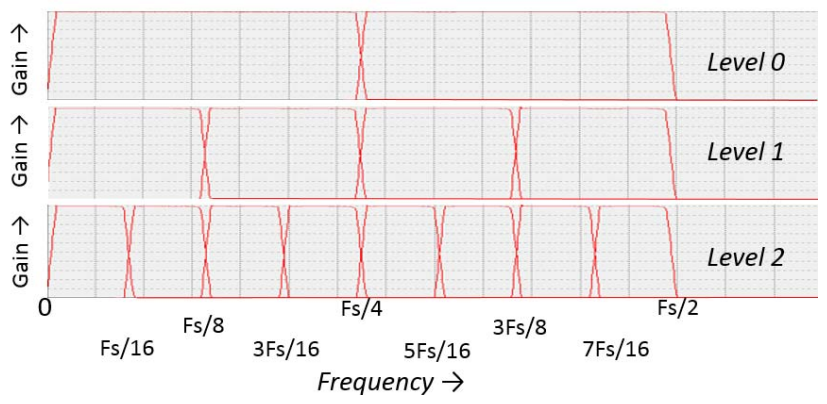

Fig. 5 Resulting frequency bands from 3 level MRA scheme.

Fig.5 show the frequency bands when implementing the method using LPFs of order 20. It can be seen that there are gaps at the band transitions which are due to the LPF not having a perfectly sharp cut-off. So any frequency component that falls in these gaps will be measured with an error. This transition error is also common to the wavelet MRA scheme.

To avoid the gaps, overlapping frequency bands for each transition can be added as an extra horizontal branch in Fig.3; this will be described at the conference.

\section{CONCLUSION}

A heterodyne based algorithm, formed by a connected structure of a single modular element is described. The modular structure is convenient to be optimised for accuracy versus processing speed. The method provides a continuous output which is ideal for measuring non-stationary waveforms and it can be made digitally equivalent to the analogue receiver specified in CISPR 16-1 [2].

Algorithm efficiency optimisations, processing speed, accuracy, output processing and implementation details for a 2-150 kHz version, together with test results will be described at the conference.

\section{ACKNOWLEDGEMENT}

This work is carried out within the EMPIR project 17NRM02 MeterEMI which has received funding from the EMPIR programme co-financed by the Participating States and from the EU's Horizon 2020 research and innovation programme.

\section{REFERENCES}

[1] Study report on electromagnetic interference between electrical equipment/systems in the frequency range below $150 \mathrm{kHz}$, CENELEC, CLC/TR 50627, 2015.

[2] Radio disturbance and immunity measuring apparatus, CISPR 16-1-1, 2019.

[3] "Heterodyne" Online: https://en.wikipedia.org/wiki/Heterodyne, Accessed 12/01/20.

[4] S.G. Mallat, A Wavelet Tour of Signal Processing, Academic Press, 1999.

[5] "A robust measurement method for supraharmonics under power frequency deviations", S. Lodetti et.al., Applied Measurements for Power Systems (AMPS), November 2019. 